\title{
TU/e emonownen

\section{Effect of exciton diffusion on the triplet-triplet annihilation rate in organic semiconductor host-guest systems}

\section{Citation for published version (APA):}

Coehoorn, R., Bobbert, P. A., \& van Eersel, H. (2019). Effect of exciton diffusion on the triplet-triplet annihilation rate in organic semiconductor host-guest systems. Physical Review B, 99(2), [024201].

https://doi.org/10.1103/PhysRevB.99.024201

DOI:

10.1103/PhysRevB.99.024201

Document status and date:

Published: 07/01/2019

\section{Document Version:}

Publisher's PDF, also known as Version of Record (includes final page, issue and volume numbers)

\section{Please check the document version of this publication:}

- A submitted manuscript is the version of the article upon submission and before peer-review. There can be important differences between the submitted version and the official published version of record. People interested in the research are advised to contact the author for the final version of the publication, or visit the $\mathrm{DOI}$ to the publisher's website.

- The final author version and the galley proof are versions of the publication after peer review.

- The final published version features the final layout of the paper including the volume, issue and page numbers.

Link to publication

\section{General rights}

Copyright and moral rights for the publications made accessible in the public portal are retained by the authors and/or other copyright owners and it is a condition of accessing publications that users recognise and abide by the legal requirements associated with these rights.

- Users may download and print one copy of any publication from the public portal for the purpose of private study or research.

- You may not further distribute the material or use it for any profit-making activity or commercial gain

- You may freely distribute the URL identifying the publication in the public portal.

If the publication is distributed under the terms of Article 25fa of the Dutch Copyright Act, indicated by the "Taverne" license above, please follow below link for the End User Agreement:

www.tue.nl/taverne

Take down policy

If you believe that this document breaches copyright please contact us at:

openaccess@tue.nl

providing details and we will investigate your claim. 


\title{
Effect of exciton diffusion on the triplet-triplet annihilation rate in organic semiconductor host-guest systems
}

\author{
R. Coehoorn, ${ }^{1, *}$ P. A. Bobbert, ${ }^{1}$ and H. van Eersel $^{2}$ \\ ${ }^{1}$ Department of Applied Physics and Institute for Complex Molecular Systems, Eindhoven University of Technology, P.O. Box 513, \\ NL-5600 MB Eindhoven, The Netherlands \\ ${ }^{2}$ Simbeyond B.V., Groene Loper 5, NL-5612 AE Eindhoven, The Netherlands
}

(Received 20 August 2018; revised manuscript received 21 December 2018; published 7 January 2019)

\begin{abstract}
We study the contribution of triplet exciton diffusion to the efficiency loss resulting from Förster-type triplet-triplet annihilation (TTA) in organic phosphorescent semiconductor host-guest systems, using kinetic Monte Carlo (KMC) simulations. Our study focusses on diffusion due to Förster-type guest-guest transfer, but includes also a comparison with simulation results for the case of Dexter-type guest-guest transfer. The simulations are carried out for a wide range of Förster radii, and for guest concentrations up to $100 \mathrm{~mol} \%$, with the purpose to support analyses of time-resolved photoluminescence experiments probing TTA. We find that the relative contribution of diffusion to the TTA-induced efficiency loss may be deduced quite accurately from a quantitative experimental measure for the shape of the time-dependent photoluminescence intensity, the so-called $r$ ratio. For small guest concentrations and Förster radii that are most relevant to organic light-emitting diodes (OLEDs), the diffusion contribution is in general quite small. Under these weak-diffusion conditions, the absolute diffusion contribution to the TTA-induced efficiency loss can be understood quantitatively using a capture radius formalism. The effective guest-guest diffusion coefficient that follows from the TTA simulations, using the capture radius formalism, agrees well with the diffusion coefficient that follows from direct KMC diffusion simulations. The simulations reveal that the diffusion coefficient is strongly affected by the randomness of the distribution of guest molecule locations.
\end{abstract}

DOI: 10.1103/PhysRevB.99.024201

\section{INTRODUCTION}

The internal quantum efficiency (IQE) of phosphorescent organic light-emitting diodes (OLEDs) shows in general a decrease with increasing current density ("roll-off"), caused by triplet-triplet annihilation (TTA) and triplet-polaron quenching (TPQ) [1-3]. The local energy dissipation that results from these two processes can also trigger molecular degradation, reducing the operational OLED device lifetime [4-6]. The development of in-depth understanding requires a multiscale approach, including the molecular scale, at which the final excitonic interaction processes take place, as well as the mesoscopic scale, at which charge and exciton diffusion take place. Whereas recently much progress has been made concerning the detailed molecular-scale description of charge transport processes [7-9], less is known about the triplet transfer, TTA and TPQ processes in phosphorescent host-guest systems. The distance $(R)$ dependence of these processes is described assuming a Förster-type algebraic $\left(R^{-6}\right)$ dependence, or a Dexter-type exponential $[\exp (-2 R / \lambda)]$ dependence, with $\lambda$ the triplet exciton wave-function decay length. The dependence of the rate of these processes on the energy difference between the two molecules involved, affecting the temperature dependence, has been described using Miller-Abrahams [10] or Marcus-type [11] transfer rates. Within a proper description of TTA and TPQ, also the positional and energetic

*r.coehoorn@tue.nl disorder of the phosphorescent guest molecules should be included.

Deriving from experiment a quantitative mechanistic picture of the interplay of all excitonic processes is in practice quite difficult. From most experimental studies carried out so far, the loss rate of the triplet volume density $(T)$ resulting from TTA is therefore expressed phenomenologically as

$$
\frac{\mathrm{d} T}{\mathrm{~d} t}=-k_{\mathrm{TT}} f T^{2},
$$

with $t$ the time, $k_{\mathrm{TT}}$ a rate coefficient, and $f$ the fraction of triplets lost in the process $(f=1 / 2$ if one triplet is lost, and $f=1$ if both triplets are lost). Similarly, the loss due to TPQ is expressed using the expression $d T / d t=-k_{\mathrm{TPQ}} n T$, with $n$ the polaron volume density. From KMC simulations, we have shown that these descriptions can indeed provide a proper description of the local rate, but not under all conditions [1214]. For Förster-type TTA, $k_{\mathrm{TT}}$ is not a constant but increases at high triplet densities [12]. Similarly, for nearest-neighbortype TPQ, $k_{\mathrm{TPQ}}$ is not a constant but increases at high polaron densities and electric fields [13]. These complications must be taken into account when analyzing steady-state experiments probing TTA and TPQ. KMC simulations have revealed a further breakdown of the validity of Eq. (1) when applied to timeresolved photoluminescence studies probing TTA [12]. In systems with only weak triplet exciton diffusion, the implicit assumption that underlies Eq. (1), viz. that the triplet volume density is spatially uniform, becomes in such experiments to an increasing degree incorrect when time proceeds. The role 
of guest-guest exciton diffusion can be probed by studying the guest concentration dependence of the time-dependent PL intensity as a function of the initial triplet volume density $[15,16]$, but also by analyzing, for a specific guest concentration, the precise shape of the time-dependent PL intensity $[12,17]$. As the latter method can already be applied to a single film composition, it is, in principle, quite powerful as a means to disentangle the roles of the direct TTA loss-process and the guest-guest diffusion process that enhances the overall TTA loss. However, such an analysis has so far only be carried out for a few specific material systems [16-18]. A wider view on the applicability of this approach is still missing.

In this paper, we present the results of a KMC simulation study of the effects of exciton diffusion on the efficiency loss due to TTA as probed in time-resolved PL studies of phosphorescent host-guest systems. Our aim is to provide a systematic and complete analysis for model materials with guest concentrations that are realistically used in OLEDs. We focus on materials in which TTA and guest-guest diffusion are both Förster-type processes. Advantageously, for a material with a given emissive lifetime only one free parameter is then needed to describe the distance-dependent rate of each process, viz. the Förster radius (see below). When assuming Dexter transfer, two parameters would be needed, viz. the rate in the zero-distance limit and the exciton wave-function decay length [20]. From experiment, a predominant role of the Dexter mechanism for guest-guest transfer at guest concentrations in the $5-15 \mathrm{~mol} \%$ range cannot be ruled out [18]. However, recent results from first-principles theory indicate that in that range Förster transfer contributes similarly to triplet exciton diffusion between the metal-organic emissive dye molecules [21]. We include in this paper a brief comparison between the contributions to the TTA loss resulting from diffusion described by both mechanisms. The simulations include the discreteness of the molecular positions, where we assume for simplicity a simple cubic lattice. The effects of diffusion are analyzed using the results of separate KMC simulations of exciton diffusion in host-guest systems. These simulations show that for host-guest systems used typically in OLEDs, with guest concentrations up to $15 \mathrm{~mol} \%$, the randomness of the locations of the guest molecules strongly affects the effective diffusion coefficient. As a result, expressions of the guest concentration dependence of the diffusion coefficient proposed earlier in the literature are in this relevant concentration range invalid.

The paper is organized as follows. In Sec. II, a brief summary is given of the method for simulating and analyzing time-resolved PL experiments probing TTA. In Sec. III, we show how for Förster-type TTA in the absence of diffusion, the time and initial triplet density dependence as probed by a time-resolved PL experiment deviates from the shape expected in the strong-diffusion limit. In Sec. IV, the effects of Förster-type diffusion on the efficiency loss and the detailed time-dependent PL intensity are discussed. We provide in this section also a brief comparison with results obtained assuming Dexter-type diffusion. Section V gives a summary and conclusions. In the Appendix, we provide support for the analysis of diffusion effects on the TTA process by giving results of KMC simulations of exciton diffusion in host-guest systems.

\section{SIMULATION AND ANALYSIS METHODS}

We adopt in this paper the method for simulating the emission intensity decay as observed from timeresolved PL experiments using the methodology described in Refs. [12,16-18]. A technical explanation of the sequence of substeps performed upon each Monte Carlo step (calculation of the rate for each possible event, event selection, event execution and simulation time update) is given in the Appendix of Ref. [12]. In short, we use the BUMBLEBEE KMC simulation tool [22] to calculate the time-dependent radiative decay $I(t)$ occurring after photon absorption at $t=0$ in a dilute hostguest system. The guest molecules are randomly positioned on the sites of a simple cubic lattice with a lattice parameter (intersite distance) equal to $a_{0}=1 \mathrm{~nm}$. The size of each system ("simulation box") considered is $100 \times 100 \times 100$ sites, with periodic boundary conditions. In the dilute host-guest systems studied experimentally, almost all absorption occurs at the host material. Fast singlet exciton transfer between the host molecules, which act as a donor, followed by fast transfer to the guest molecules, which act as an acceptor, leads almost instantaneously (within a few nanoseconds) to essentially $100 \%$ transfer to the guest molecules. Subsequently, intersystem crossing at the metal-organic phosphorescent guest molecules leads to fast singlet-to-triplet conversion. Due to the large energy difference between singlet and triplet exciton states, reverse intersystem crossing is very slow and has been neglected. In the simulations, we therefore make the simplifying assumption that the absorption process leads to the immediate generation of triplet excitons on the guest molecules. All simulations have been performed for an initial triplet volume density equal to $T_{0}=1 \times 10^{24} \mathrm{~m}^{-3}$, corresponding to 1000 triplet excitons in the simulation box. Even for a guest concentration as small as 2 mol\% (20 000 molecules in the simulation box containing $10^{6}$ molecules), only $5 \%$ of the guest molecules are thus excited at $t=0$. We find that the dependence of the TTA rate coefficients on $T_{0}$ is for values around or below the value chosen in this work very weak [17]. The triplet exciton energy of the host molecules is assumed to be much larger than that of the guest molecules, so that no triplet exciton transfer to the host molecules occurs. From experiment, we have recently found that this requires that the difference between the host and guest triplet energies is at least $0.2 \mathrm{eV}$ [18]. The output from each simulation is the set of times at which radiative decay takes place from those excitons (at most 1000) that were not lost due to TTA. From the cumulative emission curve that can be deduced from this dataset, detailed information about the nature of the TTA process is deduced in a manner discussed below. In order to improve the numerical accuracy, the number of emission events on which the analysis is based is enhanced by carrying out these simulations (in parallel or sequentially) for a large number of simulation boxes with different random positions of the guest molecules.

Triplet exciton diffusion and TTA are described as the result of a dipole-dipole (Förster-type) interaction. For the emitter molecules used in OLEDs, this is made possible by the admixture of a small amount of singlet character due to the spin-orbit coupling induced by a heavy-metal atom such as iridium (see Ref. [12] for a more extended description 
of the process). The rates are equal to $(1 / \tau)\left(R_{\mathrm{F}, \text { diff }} / R\right)^{6}$ and $(1 / \tau)\left(R_{\mathrm{F}, \mathrm{TT}} / R\right)^{6}$, with $R_{\mathrm{F}, \text { diff }}$ and $R_{\mathrm{F}, \mathrm{TT}}$ the Förster radii for the triplet exciton transfer and triplet-triplet interaction processes, respectively, and $R$ the intermolecular distance. We note that in actual materials the disordered (amorphous) structure leads to a distribution of triplet energies ("energetic disorder"). In order to limit the number of simulation parameters, this effect is neglected.

TTA is described as a process upon which a triplet on a first molecule gives rise to a higher-energy excitation of a second molecule on which already a triplet resides, after which the first molecule is left in the ground state. Upon each triplet-triplet interaction process, one of the two triplets is thus annihilated. The remaining exciton can, in principle, be a singlet, a triplet or a quintet. As we consider triplets on phosphorescent metal-organic emitters based on, e.g., iridium or platinum, such as used in OLEDs, the second molecule is then left (after partial de-excitation and fast intersystem crossing) in the lowest-energy triplet-excited state. The radiative decay time used in the simulations is $\tau=1 \mu \mathrm{s}$, a typical value for metal-organic phosphorescent emitters used in OLEDs. As the triplet-triplet interaction and triplet transfer rates depend in the same manner on $\tau$, the value of $\tau$ used does not affect the relative contribution of diffusion to the TTAinduced efficiency loss. Nonradiative decay is not included. Unless stated otherwise, only interactions of a given exciton with other excitons residing in a cube of size $2 R_{\max }$ centered around that exciton are included, with $R_{\max }$ equal to the larger of $2 R_{\mathrm{F}, \mathrm{TT}}$ or $2 R_{\mathrm{F}, \mathrm{diff}}$. For $R_{\mathrm{F}, \mathrm{TT}}=6 \mathrm{~nm}$, e.g., the interaction rates with excitons, if present, on $25^{3}-1=15624$ neighbor sites are evaluated. In Sec. III, a comparison is made with results obtained for a doubled interaction range.

For systems with strong exciton diffusion (sd), the triplet volume density $T$ remains spatially uniform during the entire time-resolved PL experiment. The conventional phenomenological expression [Eq. (1)] for describing the triplet loss rate is then applicable, and the normalized decay of the emission intensity is given by

$$
\left.\frac{I(t)}{I(0)}\right|_{\mathrm{sd}}=\frac{2}{\left(2+T_{0} k_{\mathrm{TT}, \mathrm{sd}} \tau\right) \exp (t / \tau)-T_{0} k_{\mathrm{TT}, \mathrm{sd}} \tau},
$$

with $k_{\mathrm{TT}, \mathrm{sd}}$ the rate coefficient that effectively describes the triplet loss-rate under strong-diffusion conditions. From a summation over all lattice sites, it follows that $k_{\mathrm{TT}, \mathrm{sd}} \cong$ $(16.804 / \tau) R_{\mathrm{F}, \mathrm{TT}}{ }^{6} / a_{0}{ }^{3}$. We note that the prefactor includes a factor of 2, as within the description of the process defined above the interaction rate for each triplet pair is $(2 / \tau)\left(R_{\mathrm{F}, \mathrm{TT}} / R\right)^{6}$.

However, in the case of weak exciton diffusion, the exciton loss rate due to TTA decreases more strongly with time than as expected from Eq. (1), because excitons in a nearby pair annihilate first so that the initial spatially random distribution gradually develops into a spatially correlated distribution with rather large nearest-neighbour distances. In that case, Eq. (2) is no longer applicable and the value of $k_{\mathrm{TT}}$ obtained (incorrectly) from the experimental data assuming that Eq. (2) would be valid will depend on the extraction method used. In Ref. [12], we have proposed to probe the role of triplet diffusion by comparing the value $k_{\mathrm{TT}, 1}$ that would follow using
Eq. (2) from the measured time at which half of the emission has taken place with the value $k_{\mathrm{TT}, 2}$ that would follow using Eq. (2) from the decrease of the total PL efficiency at the value of $T_{0}$ employed with respect to the PL efficiency in the zero-fluence limit. The ratio $r$ between both quantities,

$$
r \equiv \frac{k_{\mathrm{TT}, 2}}{k_{\mathrm{TT}, 1}}
$$

is equal to unity in the strong-diffusion limit, but can exceed 2 in the absence of triplet diffusion [12]. Experiments for the systems CBP:Ir(ppy $)_{2}$ (acac) [16] and TCTA:Ir(ppy $)_{2}(\mathrm{acac})$ [18], indeed, show such a variation of the $r$ ratio with varying guest concentration, reflecting a varying role of exciton diffusion. Section SI in Ref. [19] gives an explanation of the method for determining $k_{\mathrm{TT}, 1}$ and $k_{\mathrm{TT}, 2}$ from the simulations and, for comparison, from experiments.

Various authors have suggested that, within an analytical continuum theory for the no-diffusion (nd) limit, the effect of the gradual development of a spatially correlated triplet distribution on the TTA-induced triplet loss rate may be expressed as [23-26]

$$
\frac{\mathrm{d} T}{\mathrm{~d} t}=-\frac{2 \pi^{3 / 2}}{3} \frac{R_{\mathrm{F}, \mathrm{TT}}^{3}}{\sqrt{\tau t}} T^{2} .
$$

This expression was obtained by extending an analytical solution given by Förster for the time dependence of the density of excited donor molecules in a system containing quenching acceptor molecules [27]. However, Zhang and Forrest have pointed out that in the derivation the time-dependence of the triplet density was not consistently included [15]. We furthermore note that (i) implicitly both interacting triplets are assumed to be lost in the process, whereas it is commonly assumed that only one triplet is lost (as in our simulations), and that (ii) for actual molecular materials a theory taking the discreteness of the molecular positions into account would be more appropriate, instead of a continuum theory. In the next section, we, nevertheless, find that for the values of $R_{\mathrm{F}, \mathrm{TT}}$ included in our study (up to $6 \mathrm{~nm}$ ), a fair description of the KMC simulation results in the no-diffusion limit is obtained from Eq. (4) after empirically modifying the TTA-induced loss rate by a factor $b \cong 0.7$. The normalized decay of the emission intensity is then given by

$$
\left.\frac{I(t)}{I(0)}\right|_{\mathrm{nd}} \cong \frac{\exp (-t / \tau)}{1+(2 / 3) b \pi^{2} R_{\mathrm{F}, \mathrm{TT}^{3}} T_{0} \operatorname{erf} \sqrt{t / \tau}},
$$

with erf the error function.

\section{FÖRSTER-TYPE TTA IN THE ABSENCE OF DIFFUSION}

Figure 1(a) shows the dependence of the total PL efficiency, $\eta_{\mathrm{PL}}$, on $R_{\mathrm{F}, \mathrm{TT}}$, as obtained from $\mathrm{KMC}$ simulations for $T_{0}=1 \times 10^{24} \mathrm{~m}^{-3}$ in the absence of diffusion. In addition to the result obtained from our default simulation approach ( $R_{\max }=2 R_{\mathrm{F}, \mathrm{TT}}$, see the previous section), also results for a doubled largest interaction distance are displayed. In terms of the fraction $\left(1-\eta_{\mathrm{PL}}\right)$ of quenched excitons, the two results differ by about $8 \%$ for $R_{\mathrm{F}, \mathrm{TT}}=3.5 \mathrm{~nm}$ (a typical experimental value) to about $2.5 \%$ for $R_{\mathrm{F}, \mathrm{TT}}=6 \mathrm{~nm}$. As these differences 

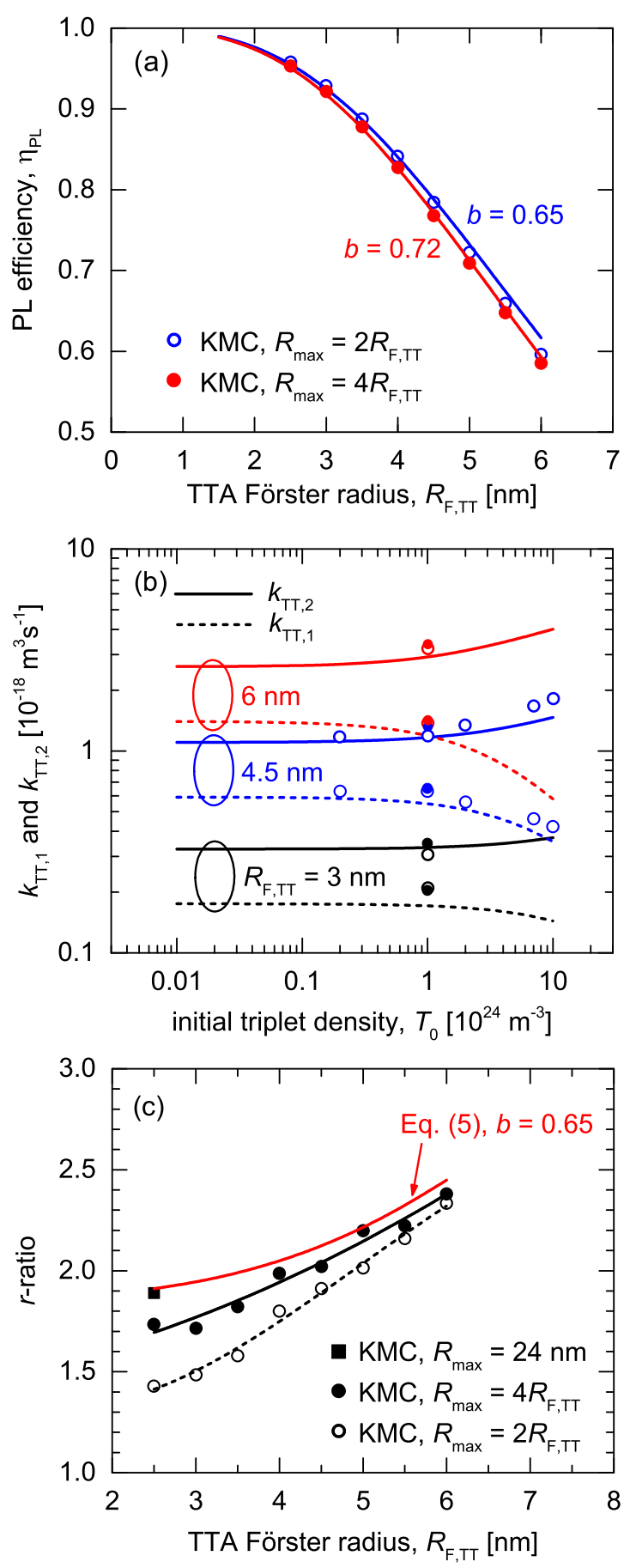

FIG. 1. KMC simulation results of Förster-type TTA in the absence of diffusion. (a) Dependence of the total PL efficiency, $\eta_{\mathrm{PL}}$, on $R_{\mathrm{F}, \mathrm{TT}}$, for two values of $R_{\max }$ (symbols), for $T_{0}=1 \times 10^{24} \mathrm{~m}^{-3}$. The curves give the results for $b=0.65$ (blue) and 0.72 (red). (b) $T_{0}$ dependence of $k_{\mathrm{TT}, 1}$ and $k_{\mathrm{TT}, 2}$ (dashed and full curves) as obtained for various values of $R_{\mathrm{F}, \mathrm{TT}}$ from Eq. (5) with $b=0.65$, and KMC simulation data for $T_{0}=1 \times 10^{24} \mathrm{~m}^{-3}$. (c). Dependence of the $r$ ratio on $R_{\mathrm{F}, \mathrm{TT}}$ for $T_{0}=1 \times 10^{24} \mathrm{~m}^{-3}$, as obtained from KMC simulations (see also Table S1 in Ref. [19]) and from Eq. (5) with $b=0.65$.

are small in comparison to typical experimental uncertainties, we regard our default approach as a fair compromise between accuracy and computational efficiency.
For the default approach, $R_{\max }=2 R_{\mathrm{F}, \mathrm{TT}}$, a good fit to the data is obtained using $b=0.65$ (blue curve in Fig. 1). For $R_{\max }=4 R_{\mathrm{F}, \mathrm{TT}}$, a good fit is obtained using $b=0.72$ (red curve). The rate coefficient $k_{\mathrm{TT}, 2}$ follows from $\eta_{\mathrm{PL}}$ via

$$
\eta_{\mathrm{PL}}\left(T_{0}\right)=-\frac{2 \ln \left(\frac{2}{2+T_{0} k_{\mathrm{TT}, 2} \tau}\right)}{T_{0} k_{\mathrm{TT}, 2} \tau} .
$$

We find that in the no-diffusion limit, the dependence of $k_{\mathrm{TT}, 2}$ on $R_{\mathrm{F}, \mathrm{TT}}$ is for the range of values of $R_{\mathrm{F}, \mathrm{TT}}$ included in this study (up to $6 \mathrm{~nm}$ ) accurately described by

$$
k_{\mathrm{TT}, 2, \mathrm{nd}}=p_{1}{\frac{R_{\mathrm{F}, \mathrm{TT}}}{\tau}}^{3}+p_{2}{\frac{R_{\mathrm{F}, \mathrm{TT}}}{\tau}}_{\tau}^{6} T_{0} .
$$

This expression is analogous to the first two terms of a series expansion in $R_{\mathrm{F}, \mathrm{TT}}{ }^{3} T_{0}$ of the rate coefficient $k_{\mathrm{TT}, \mathrm{ss}}$ for TTA under steady-state (ss) conditions and in the absence of diffusion (Eq. (7) in Ref. [12]), for which $p_{1, \mathrm{ss}}=4 \pi^{2} / 3=13.2$ and $p_{2, \mathrm{ss}}=4 \pi^{2}(\pi / 2-1) / 3=7.5$. A fit to the simulation data for $R_{\max }=2 R_{\mathrm{F}, \mathrm{TT}}\left(4 R_{\mathrm{F}, \mathrm{TT}}\right)$ leads to $p_{1}=12.2(13.2)$ and $p_{2}=14.6(11.3)$. This comparison shows that $k_{\mathrm{TT}, 2}$ as obtained from transient PL experiments is very close to the effective TTA-rate coefficient $k_{\mathrm{TT}, \mathrm{ss}}$ that determines the efficiency loss in, e.g., OLEDs under steady-state conditions, i.e., at a constant triplet density $T=T_{0}$, provided that the average density of excitons within a sphere with radius equal to $R_{\mathrm{F}, \mathrm{TT}}$ is much less than 1 . For $R_{\mathrm{F}, \mathrm{TT}}=3.5 \mathrm{~nm}$, a typical experimental value, this condition is quite well obeyed for $T_{0}=10^{24} \mathrm{~m}^{-3}$. For $R_{\mathrm{F}, \mathrm{TT}}=5 \mathrm{~nm}, k_{\mathrm{TT}, \mathrm{ss}}$ is then about a factor 1.35 larger than $k_{\mathrm{TT}, 2}$ (see Fig. S4 in Ref. [17]).

Figure 1(b) shows the $T_{0}$-dependence of $k_{\mathrm{TT}, 1}$ and $k_{\mathrm{TT}, 2}$ (dashed and full curves) as obtained using Eq. (5) with $b=$ 0.65 , and the values obtained from the KMC simulations with $R_{\max }$ equal to $2 R_{\mathrm{F}, \mathrm{TT}}$ and $4 R_{\mathrm{F}, \mathrm{TT}}$. From Eq. (5), $k_{\mathrm{TT}, 2}$ is expected to be significantly larger than $k_{\mathrm{TT}, 1}$, in agreement with the simulation results. An increase and decrease, respectively, of $k_{\mathrm{TT}, 2}$ and $k_{\mathrm{TT}, 1}$ is found beyond a certain value of $T_{0}$, as expected for $k_{\mathrm{TT}, 2}$ from Eq. (7). Such an effect was already found for TTA under static conditions. The simulation data for $R_{\mathrm{F}, \mathrm{TT}}=4.5 \mathrm{~nm}$ show that it is also found under transient conditions. The effect occurs when at $t=0$ within a sphere with radius $R_{\mathrm{F}, \mathrm{TT}}$ on average more than one exciton is present [12].

Figure 1(c) shows that from Eq. (5) and from the KMC simulations, the $r$ ratio increases with increasing $R_{\mathrm{F}, \mathrm{TT}}$. This dependence is caused by the increase and decrease of $k_{\mathrm{TT}, 2}$ and $k_{\mathrm{TT}, 1}$, respectively, with increasing $T_{0}$, which for a given value of $T_{0}$ is largest for large $R_{\mathrm{F}, \mathrm{TT}}$ [see Fig. 1(b)]. In the limit of small $T_{0}$, the dependence of the $r$ ratio on $R_{\mathrm{F}, \mathrm{TT}}$ vanishes. Eq. (5) yields then $r=1.87$, almost independent of the precise value of $b$ in the range $0.65-0.75$. With increasing $R_{\max }$, the simulation curves approach the theoretical curve, obtained from Eq. (5) with $b=0.65$. The difference is largest for $R_{\mathrm{F}, \mathrm{TT}}=2.5 \mathrm{~nm}$, for which the two data series shown are based on values of $R_{\max }$ equal to 5 and $10 \mathrm{~nm}$. For $R_{\max }=24 \mathrm{~nm}$ (squared symbol, 117648 neighbours included), the $r$ ratio as obtained from the simulations is essentially equal to the value of 1.91 obtained from Eq. (5). The dependence of the 
simulation results on $R_{\max }$ is mainly due to an increase of $k_{\mathrm{TT}, 2}$ with increasing $R_{\max }$, because it increases with decreasing PL efficiency [see Fig. 1(a)]. Within the numerical accuracy, $k_{\mathrm{TT}, 1}$ was found to be quite insensitive to $R_{\max }$. We note that the numerical accuracy of $k_{\mathrm{TT}, 2}$ is much better than that of $k_{\mathrm{TT}, 1}$. Obtaining $k_{\mathrm{TT}, 1}$ with good accuracy requires averaging over a larger number of simulations, as it is determined by the difference between the actual halftime of the cumulative emission and the halftime $\tau \times \ln 2$ that would be obtained in the absence of TTA. The simulation data shown were typically based on the results of 1000 to 2000 simulations, leading to a numerical accuracy of $k_{\mathrm{TT}, 2}$ better than $\pm 0.5 \%$ and of $k_{\mathrm{TT}, 1}$ of about $\pm 10 \%$ for $R_{\mathrm{FTT}}=2.5 \mathrm{~nm}$ to about $\pm 5 \%$ for $R_{\mathrm{F}, \mathrm{TT}}=6 \mathrm{~nm}$.

\section{EFFECTS OF DIFFUSION ON THE TTA RATE}

We have studied the effects of Förster-type diffusion using KMC simulations for values of $R_{\mathrm{F}, \mathrm{TT}}$ in the range $2.5-6 \mathrm{~nm}$, combined with values of $R_{\mathrm{F} \text {,diff }}$ in the range $2-3.5 \mathrm{~nm}$, for guest concentrations $c_{\mathrm{g}}=1$ to $100 \mathrm{~mol} \%$, with $T_{0}=10^{24} \mathrm{~m}^{-3}$ and $R_{\max }=2 R_{\mathrm{F}, \mathrm{TT}}$. At the end of subsection $\mathrm{A}$, a brief comparison is given with KMC simulation results obtained assuming Dexter transfer. Sections SII and SIII in Ref. [19] contain tabulated overviews of the simulation results, in total for 156 systems.

\section{A. Effect of diffusion on $\boldsymbol{k}_{\mathrm{TT}, 2}$}

Figures 2(a) and 2(b) show the guest concentration dependence of $k_{\mathrm{TT}, 2}$, obtained for various values of $R_{\mathrm{F} \text {,diff, for }}$ $R_{\mathrm{F}, \mathrm{TT}}=3.5$ and $5 \mathrm{~nm}$, respectively. As expected, the effect of diffusion increases with increasing $c_{\mathrm{g}}$ and $R_{\mathrm{F} \text {,diff }}$. However, this sensitivity is for $c_{\mathrm{g}}$ close to $100 \mathrm{~mol} \%$ smaller when $R_{\mathrm{F}, \mathrm{TT}}=3.5 \mathrm{~nm}$ than when $R_{\mathrm{F}, \mathrm{TT}}=5 \mathrm{~nm}$. This may be explained by considering the effect of diffusion on TTA. The effect on $k_{\mathrm{TT}, 2}$ of a change of the triplet diffusion coefficient $D$ is large in the intermediate-diffusion regime, when exciton diffusion significantly but not yet completely eliminates the spatially correlated disorder of exciton positions. However, a change of $D$ does not affect $k_{\mathrm{TT}, 2}$ anymore in the strongdiffusion regime. For systems with a small value of $R_{\mathrm{F}, \mathrm{TT}}$, the strong-diffusion regime is more easily reached, as diffusion over only a small distance will already adequately eliminate the correlated disorder of exciton positions. That may be seen more clearly from Fig. 2(c), which shows how for a given value of $R_{\mathrm{F}, \mathrm{TT}}$ the rate coefficient increases with the guest concentration, until saturation takes place when the strongdiffusion limit is reached. From experiment, we regard values of $R_{\mathrm{F}, \mathrm{TT}} \cong 3.5 \mathrm{~nm}$ and $R_{\mathrm{F} \text {,diff }} \cong 2 \mathrm{~nm}$ as realistic for host-guest systems used in OLEDs. Figure 2(a) shows that for guest concentrations that are relevant to OLED applications, around or below $10 \mathrm{~mol} \%$, the effect of diffusion on the TTA loss is weak or intermediate; the strong-diffusion regime is not reached.

In Fig. 3, we focus on $k_{\mathrm{TT}, 2}$ in the application-relevant low-concentration range. The figure shows that for all values of $R_{\mathrm{F}, \mathrm{TT}}$ and $R_{\mathrm{F}, \mathrm{diff}}$ included in the simulations, $k_{\mathrm{TT}, 2}$ varies to an excellent approximation linearly with $c_{\mathrm{g}}$. The slope of these curves, which gives the sensitivity of $k_{\mathrm{TT}, 2}$ to a con-
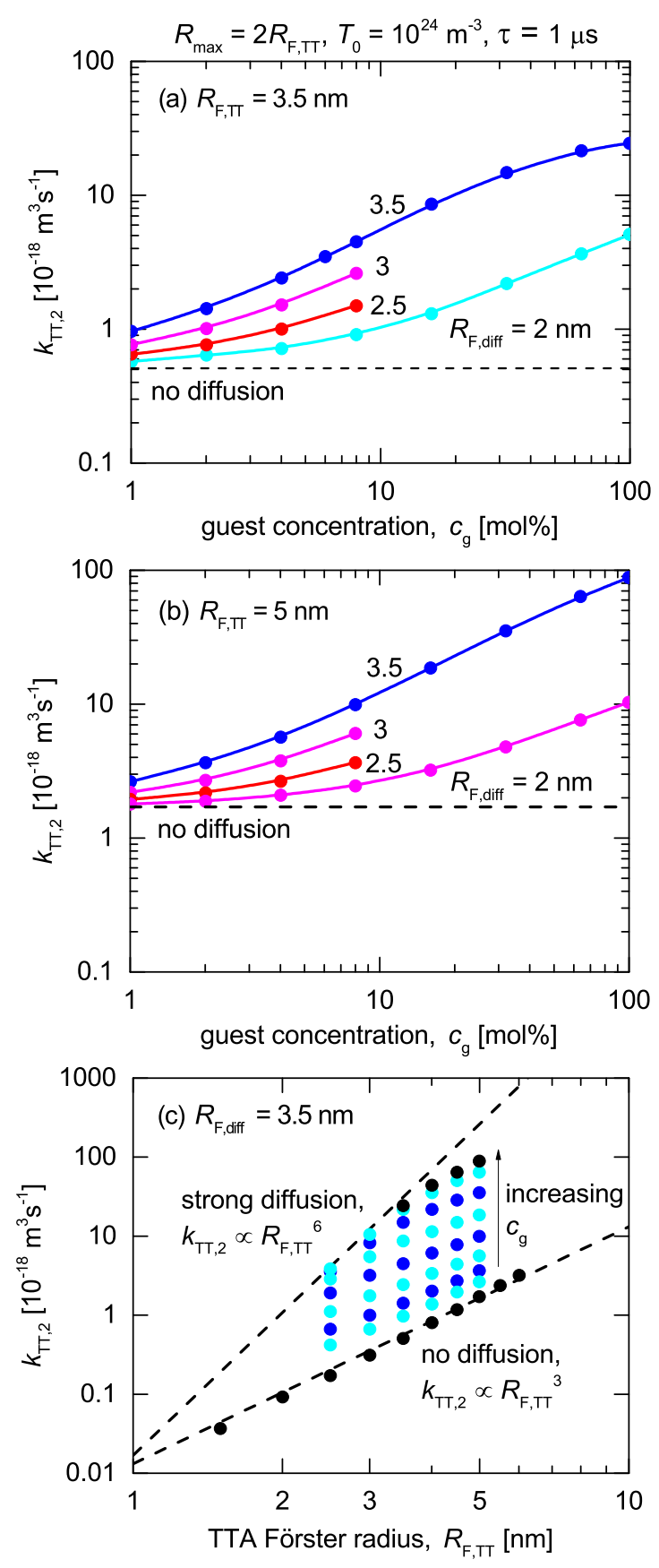

FIG. 2. KMC simulation results of the rate coefficient $k_{\mathrm{TT}, 2}$ describing the PL efficiency loss due to Förster-type TTA combined with Förster-type diffusion. [(a) and (b)] Dependence on the guest concentration and $R_{\mathrm{F}, \mathrm{diff}}$, for $R_{\mathrm{F}, \mathrm{TT}}=3.5 \mathrm{~nm}$ and $R_{\mathrm{F}, \mathrm{TT}}=5 \mathrm{~nm}$, respectively. (c) Dependence on $R_{\mathrm{F}, \mathrm{TT}}$ for $R_{\mathrm{F} \text {,diff }}=3.5 \mathrm{~nm}$, for (from bottom to top) the following conditions: no diffusion, $c_{\mathrm{g}}=1,2,4$, $8,16,32,64$, and (only for $R_{\mathrm{F}, \mathrm{TT}}=3.5,4$, and $4.5 \mathrm{~nm}$ ) $100 \mathrm{~mol} \%$. The dashed strong-diffusion line gives $k_{\mathrm{TT}, \mathrm{sd}}$ (see Sec. II) and the dashed no-diffusion line gives $k_{\mathrm{TT}, 2, \text { nd }}$ as obtained from Eq. (7) with (for simplicity) $p_{1}=4 \pi^{2} / 3$ and $p_{2}=0$.

centration change, increases with increasing $R_{\mathrm{F}, \mathrm{TT}}$ and $R_{\mathrm{F} \text {,diff }}$. Figure 4 shows that this sensitivity varies for a given value of $R_{\mathrm{F}, \text { diff }}$ linearly with $R_{\mathrm{F}, \mathrm{TT}}$, and approaches zero when $R_{\mathrm{F}, \text { diff }}$ approaches a cutoff value $R_{0}=1.8 \mathrm{~nm}$. For the concentration 


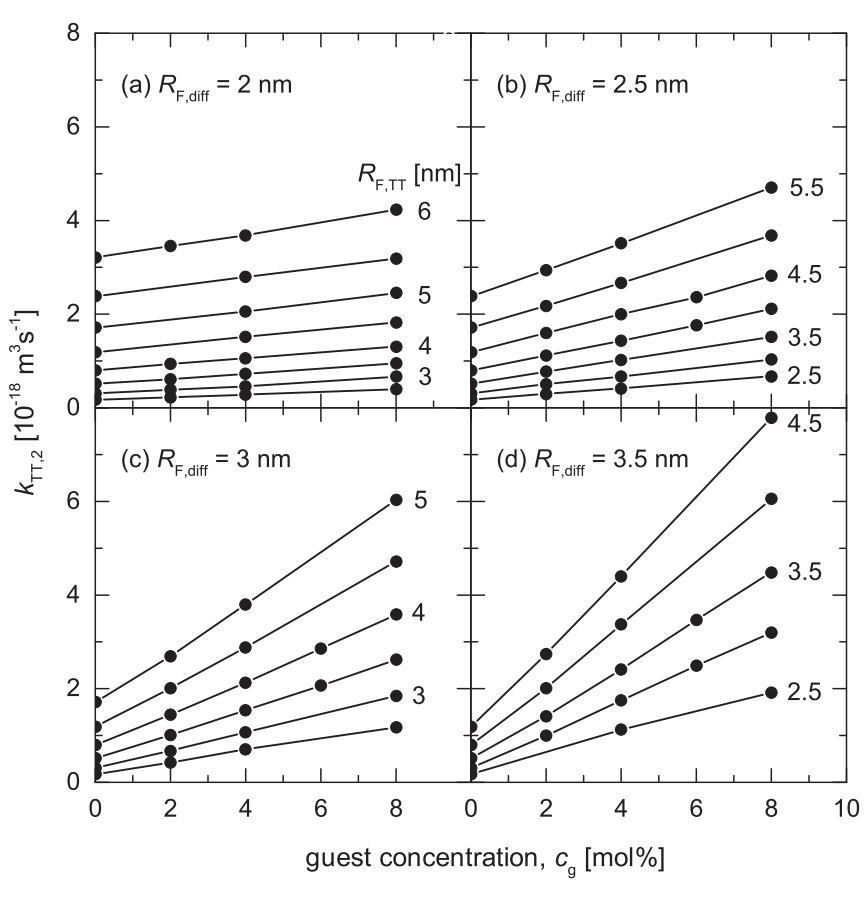

FIG. 3. Dependence of the rate coefficient $k_{\mathrm{TT}, 2}$ on the guest concentration for various values of $R_{\mathrm{F}, \mathrm{TT}}$ and for $R_{\mathrm{F}, \mathrm{diff}}=2$ (a), 2.5 (b), 3 (c) and $3.5 \mathrm{~nm}$ (d), from KMC simulations for $T_{0}=$ $10^{24} \mathrm{~m}^{-3}$ and $\tau=1 \mu \mathrm{s}$. The lines give a linear interpolation between successive data points.

range shown ( $\left.c_{\mathrm{g}} \leqslant 8 \mathrm{~mol} \%\right)$, the effect of diffusion is to a very good approximation additive, so that $k_{\mathrm{TT}, 2}$ can be described as a sum of $k_{\mathrm{TT}, 2, \text { nd }}$ plus a diffusion contribution $k_{\mathrm{TT}, 2, \mathrm{diff}}$ :

$$
\begin{aligned}
k_{\mathrm{TT}, 2} & =k_{\mathrm{TT}, 2, \mathrm{nd}}+k_{\mathrm{TT}, 2, \mathrm{diff}} \\
& =k_{\mathrm{TT}, 2, \mathrm{nd}}+p_{3} \frac{\left(R_{\mathrm{F}, \mathrm{TT}}-R_{0}\right)}{a_{0}}\left(\frac{R_{\mathrm{F}, \mathrm{diff}}}{a_{0}}\right)^{4.1} c_{\mathrm{g}},
\end{aligned}
$$

with $p_{3}=1.8 \times 10^{-21} \mathrm{~m}^{3} \mathrm{~s}^{-1}(\mathrm{~mol} \%)^{-1}$ if $c_{\mathrm{g}}$ is expressed in units $\mathrm{mol} \% . R_{0}$ is independent of all other parameters and

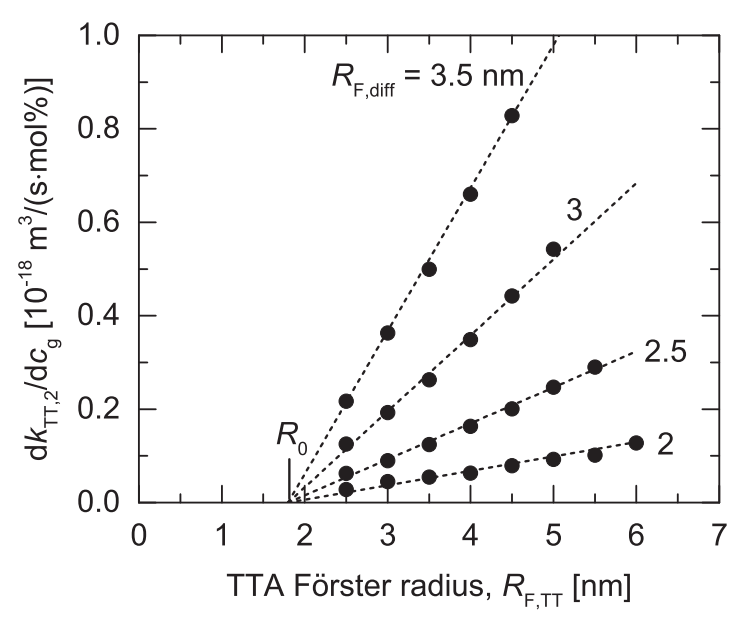

FIG. 4. Sensitivity of $k_{\mathrm{TT}, 2}$ to the guest concentration as a function of $R_{\mathrm{F}, \mathrm{TT}}$, obtained from KMC simulations for various values of $R_{\mathrm{F}, \mathrm{diff}}$ (symbols). The dashed lines give a fit using Eq. (8). The sensitivity vanishes for $R_{\mathrm{F}, \mathrm{TT}}=R_{0}=1.8 \mathrm{~nm}$. equal to the 1.8-nm cutoff distance mentioned above. Figure 4 shows that the quality of the fit is excellent.

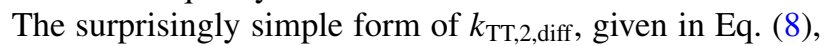
may be rationalized as follows. Within a capture radius formalism [15], one expects that the diffusion contribution may be expressed as

$$
k_{\mathrm{TT}, 2, \mathrm{diff}} \cong 8 \pi R_{\mathrm{c}, \mathrm{eff}} D,
$$

with $R_{\text {c,eff }}$ an effective capture radius and $D$ the triplet exciton diffusion coefficient. The simulation results suggest that the effective capture radius is equal to

$$
R_{\mathrm{c}, \text { eff }}=R_{\mathrm{F}, \mathrm{TT}}-R_{0} .
$$

Explicit KMC simulations of the diffusion coefficient, presented in Appendix, show that for small guest concentrations the diffusion coefficient is due to the randomness of the guest molecule positions enhanced over the value that would be expected when assuming that the guest molecules reside on an ordered lattice of sites. Consistent with Eq. (8), the diffusion coefficient depends for $c_{\mathrm{g}}$ in the $2-5 \mathrm{~mol} \%$ range approximately linearly on $c_{\mathrm{g}}$ [Fig. $8(\mathrm{a})$ ]. Furthermore, $D$ is approximately proportional to $R_{\mathrm{F}, \mathrm{diff}}{ }^{\alpha}$, with $\alpha$ in the range 4-5 ([Fig. 8(b)]. This finding provides a rationalization for the empirical exponent of 4.1 given in Eq. (8). As a representative example, we consider a system with $c_{\mathrm{g}}=5 \mathrm{~mol} \%$, $R_{\mathrm{F}, \mathrm{TT}}=3.5 \mathrm{~nm}$ and $R_{\mathrm{F} \text {,diff }}=2 \mathrm{~nm}$. Neglecting the diffusion contribution, $k_{\mathrm{TT}, 2 \text {,diff }}$ would [from Eq. (7)] then be equal to $0.56 \times 10^{-18} \mathrm{~m}^{3} \mathrm{~s}^{-1}$. From Eq. (8), the value of $k_{\mathrm{TT}, 2, \mathrm{diff}} \mathrm{ob}-$ tained from the simulations is $0.79 \times 10^{-18} \mathrm{~m}^{3} \mathrm{~s}^{-1}$, whereas using Eqs. (9) and (10) the value of $k_{\mathrm{TT}, 2, \mathrm{diff}}$ estimated from the capture radius formalism is $0.48 \times 10^{-18} \mathrm{~m}^{3} \mathrm{~s}^{-1}$. In view of the simplifications made, we consider the agreement as satisfactory.

As discussed in Introduction, we cannot exclude that in phosphorescent metal-organic host-guest systems used in OLEDs Dexter-type exciton transfer contributes at high concentrations more strongly to the diffusion coefficient than Förster-type transfer. For large values of the Dexter rate in the zero-distance limit, $k_{\mathrm{D}, 0}$, and for a realistic value of the wave-function decay length, $\lambda=0.3 \mathrm{~nm}$, the dependence of the diffusion coefficient on the guest concentration is stronger than as obtained assuming Förster-type transfer, as shown in Sec. SIV in Ref. [19]. The diffusion contribution to $k_{\mathrm{TT}, 2}$ shows for the case of Dexter transfer therefore a superlinear dependence on the guest concentration dependence (see Sec. $\mathrm{SV}$ in Ref. [19]). For $R_{\mathrm{F}, \mathrm{TT}} \geqslant 3 \mathrm{~nm}$, the diffusion contribution is found to be approximately proportional to $\left(R_{\mathrm{F}, \mathrm{TT}}-R_{0}\right)$, with $R_{0}=1.8 \mathrm{~nm}$, as for the case of Förster transfer. For a system with $c_{\mathrm{g}}=20 \mathrm{~mol} \%, R_{\mathrm{F}, \mathrm{TT}}=4 \mathrm{~nm}, k_{\mathrm{D}, 0}=1.6 \times 10^{11} \mathrm{~s}^{-1}$, and $\lambda=0.3 \mathrm{~nm}, k_{\mathrm{TT}, 2 \text {,diff }}$ is equal to $1.00 \times 10^{-18} \mathrm{~m}^{3} \mathrm{~s}^{-1}$. For comparison, $k_{\mathrm{TT}, 2, \mathrm{nd}}$ is then equal to $0.84 \times 10^{-18} \mathrm{~m}^{3} \mathrm{~s}^{-1}$. Using Eqs. (9) and (10), the value estimated from the capture radius formalism assuming $R_{\mathrm{c}, \text { eff }}=R_{\mathrm{F}, \mathrm{TT}}-R_{0}$ would be $0.51 \times 10^{-18} \mathrm{~m}^{3} \mathrm{~s}^{-1}$. The capture radius formalism thus provides again a good rough estimate.

\section{B. Effect of diffusion on the $r$ ratio}

In the previous subsection, we have concluded that for Förster radii and guest concentrations that are relevant 


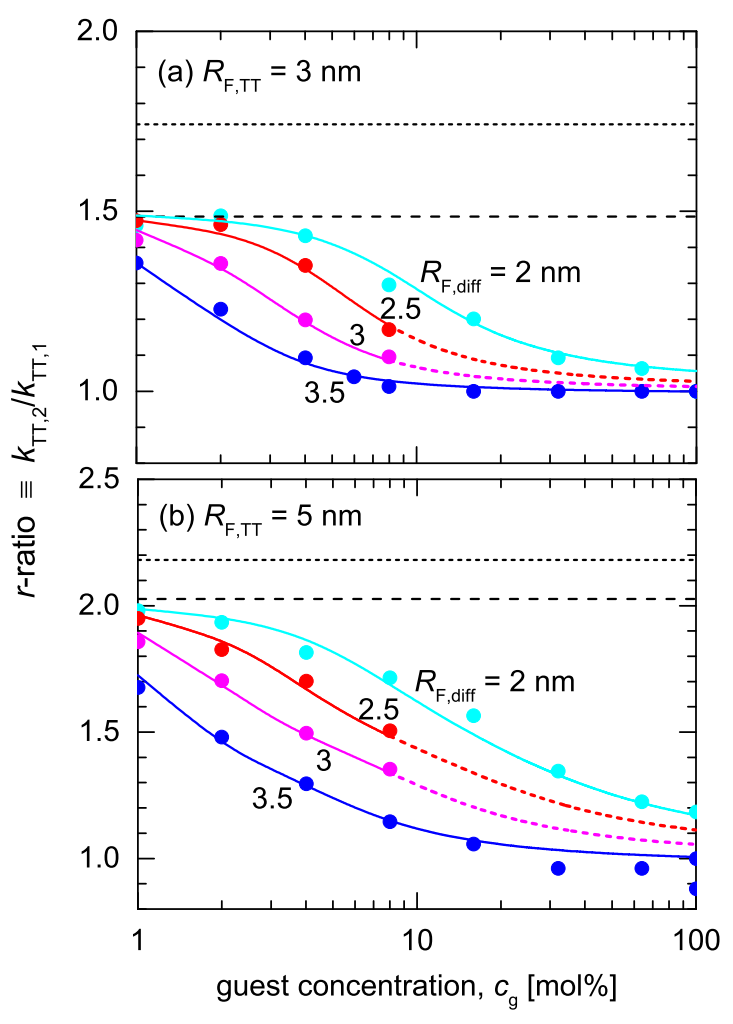

FIG. 5. Dependence of the $r$ ratio on the guest concentration, for various values of $R_{\mathrm{F}, \text { diff }}$ and for $R_{\mathrm{TT}, 2}=3$ (a) and $5 \mathrm{~nm}$ (b). The full curves (and their dashed extrapolation) provide a guide to the eye. The long-dashed (short-dashed) horizontal lines give the $r$ ratio in the absence of diffusion, obtained from simulations with $R_{\max }=$ $2 R_{\mathrm{TT}, 2}\left(4 R_{\mathrm{TT}, 2}\right)$. The numerical $2 \sigma$ accuracy is approximately \pm 0.1 .

to OLED applications, the strong-diffusion regime is not reached. Even for $R_{\mathrm{F}, \mathrm{TT}}$ as small as $2.5 \mathrm{~nm}$ and $R_{\mathrm{F} \text {,diff }}$ as large as $3.5 \mathrm{~nm}$, saturation of the TTA loss was only found for guest concentrations larger than about $20 \mathrm{~mol} \%$ [see Fig. 2(c)]. As discussed in Sec. II, this can be probed experimentally by measuring the $r$ ratio [Eq. (3)]. Figure 5 gives the guest concentration dependence of the $r$ ratio for $R_{\mathrm{F}, \mathrm{TT}}=3$ and $5 \mathrm{~nm}$, and for various values of $R_{\mathrm{F} \text {,diff. The decrease of the }}$ $r$ ratio with increasing guest concentration is indeed seen to reflect the gradual transition from the no-diffusion to the strong-diffusion regime.

Figure 6 shows that for all systems studied, the relative diffusion contribution to $k_{\mathrm{TT}, 2}$ is strongly correlated with the relative $r$ ratio enhancement. This remarkable result implies that for any system of the type studied in this work, the relative contribution of diffusion to $k_{\mathrm{TT}, 2}$ (and hence to the PL efficiency loss) can simply be derived from a measurement of the $r$ ratio for that system and the value $r_{\text {nd }}$ in the low-concentration (no-diffusion) limit. A simple empirical fit to the simulation data (dashed curve in the figure) has the form

$$
\frac{r-1}{r_{\mathrm{nd}}-1} \cong 1-\tanh \left[1.5 \log _{10}\left(\frac{k_{\mathrm{TT}, 2}}{k_{\mathrm{TT}, 2, \mathrm{nd}}}\right)\right]
$$

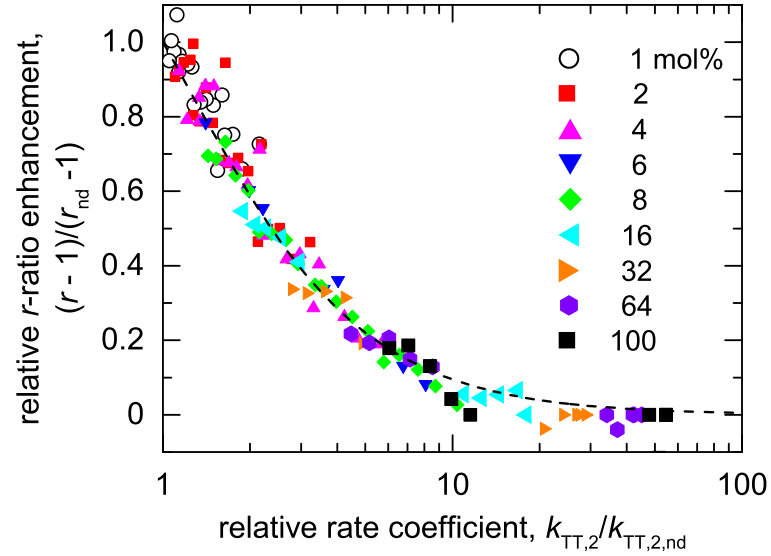

FIG. 6. Relative enhancement of the $r$ ratio with respect to the value in the strong-diffusion, normalized to the enhancement for the no-diffusion limit, $(r-1) /\left(r_{\mathrm{nd}}-1\right)$, as a function of the enhancement of $k_{\mathrm{TT}, 2}$ due to diffusion, $k_{\mathrm{TT}, 2} / k_{\mathrm{TT}, 2, \mathrm{nd}}$, as obtained from KMC simulations for all systems included in this study. The dashed curve gives an empirical fit [see Eq. (11)].

\section{SUMMARY AND CONCLUSIONS}

Using KMC simulations, we have studied how the efficiency loss due to Förster-type TTA in organic semiconductor host-guest systems is enhanced due to Förster-type guestguest diffusion. The simulations show that for realistic Förster radii describing the rates of these processes, about 3.5 and $2 \mathrm{~nm}$, respectively, and for guest concentrations around or below $10 \mathrm{~mol} \%$, as used in OLEDs, the effect of exciton diffusion can be significant, viz. up to a factor of 2 [see Fig. 2(a)]. However, in a more formal manner, the role of diffusion is still best characterized as "weak," in the sense that the diffusion of excitons is insufficient to maintain in systems in which the exciton density is locally reduced due to TTA at any moment in time still a spatially almost uniform triplet density distribution. Conventional analysis methods for analyzing the results of time-dependent PL experiments that probe TTA, assuming strong-diffusion conditions and based on Eq. (2), are therefore invalid.

We find that the effect of diffusion on the TTA-induced efficiency loss in time-dependent PL experiments, as expressed by the rate coefficient $k_{\mathrm{TT}, 2}$, can be quite accurately understood using a simple expression [Eq. (8)] within which the diffusion contribution is additive and proportional to the guest concentration, superlinearly dependent on $R_{\mathrm{F}, \mathrm{diff}}$ and proportional to an effective capture radius, $R_{\mathrm{F}, \mathrm{TT}}-R_{0}$, with $R_{0}$ a cutoff distance. The effective triplet exciton diffusion coefficient that follows from this expression using the capture radius formalism [Eq. (9)] agrees quite well with the actual triplet diffusion coefficient, obtained in Appendix for a range of concentrations and values of $R_{\mathrm{F}, \mathrm{diff}}$ from KMC simulations. Carrying out such dedicated KMC diffusion simulations was found to be necessary, as for dilute systems with random emitter locations percolating filamentary pathways lead to a strong enhancement of the diffusion coefficient as compared to the value expected from analytical theory based on the average guest-guest distance. Experimentally, the validity of the assumption of strong diffusion can be probed by analyzing 
the detailed time-dependence of the photoluminescence. We have shown how a characteristic number that follows from the precise shape of the time-dependent photoluminescence, the $r$ ratio, decreases gradually with increasing guest concentration and can be used to quantify [via Fig. 6 and Eq. (11)] the relative contribution of exciton diffusion to $k_{\mathrm{TT}, 2}$.

Future extensions of this work are expected to include the effects of molecular-scale details of the amorphous thin film materials, such as triplet energetic disorder and molecular orientational disorder, on the excitonic interaction rates. Also when such refinements are included, we expect that the methodology discussed in this paper will be a powerful tool for analyzing experiments that probe the efficiency loss due to TTA.

\section{ACKNOWLEDGMENTS}

This work was supported by the Horizon-2020 EU project MOSTOPHOS (Project No. 646259, RC, PAB). Part of the work was carried out at the Philips Research Laboratories, Eindhoven, The Netherlands (RC and HvE).

\section{APPENDIX: KMC SIMULATIONS OF EXCITON DIFFUSION IN ORGANIC HOST-GUEST SYSTEMS}

In this appendix, we use KMC simulations to study the diffusion coefficient, $D$, resulting from guest-guest exciton transfer in organic host-guest systems. We thus assume that the excitons are well-confined to the guest sites. We focus on the dependence on the guest concentration. In the main text, the results obtained in this appendix are used to analyze the guest concentration dependence of the efficiency loss due to TTA.

Approximate expressions for weakly diluted systems. For the case of nearest-neighbor $(\mathrm{NN})$ exciton transfer with a rate $k$ in a nondiluted system with all molecules positioned on a simple cubic lattice with lattice constant $a_{0}$, the diffusion coefficient is given by

$$
D_{\mathrm{NN}}=a_{0}^{2} k .
$$

We will show below from KMC simulations that for weakly diluted systems, $a_{0}$ may be replaced by the average guestguest distance $a \equiv c_{\mathrm{g}}{ }^{-1 / 3} a_{0}$, with $c_{\mathrm{g}}$ the guest concentration. For the case of Förster-type transfer, with $\tau$ the exciton lifetime and $R_{\mathrm{F}, \text { diff }}$ the Förster radius for exciton diffusion, a summation over contributions from hops over all possible lattice distances leads then to the following approximate expression for the diffusion coefficient:

$$
D \cong c_{\mathrm{sc}, \mathrm{F}} \frac{a^{2}}{\tau}\left(\frac{R_{\mathrm{F}, \mathrm{diff}}}{a}\right)^{6}
$$

with $c_{\mathrm{sc}, \mathrm{F}}=2.755$ (Ref. [28] gives $c_{\mathrm{sc}, \mathrm{F}}=2.76$ ). We note that this approximation was given earlier by Zhang and Forrest, albeit including only $\mathrm{NN}$ transfer (i.e., with $c_{\mathrm{sc}, \mathrm{F}}$ equal to 1 ) [15]. Equation (A2), within which the effects of the random distribution of emitter molecule locations are neglected, is exact in the nondilute limit. We will show below that it is not valid for strongly diluted phosphorescent host-guest systems with triplet exciton transfer parameters that are realistic for OLED applications. At small guest concentrations, Eq. (A2)

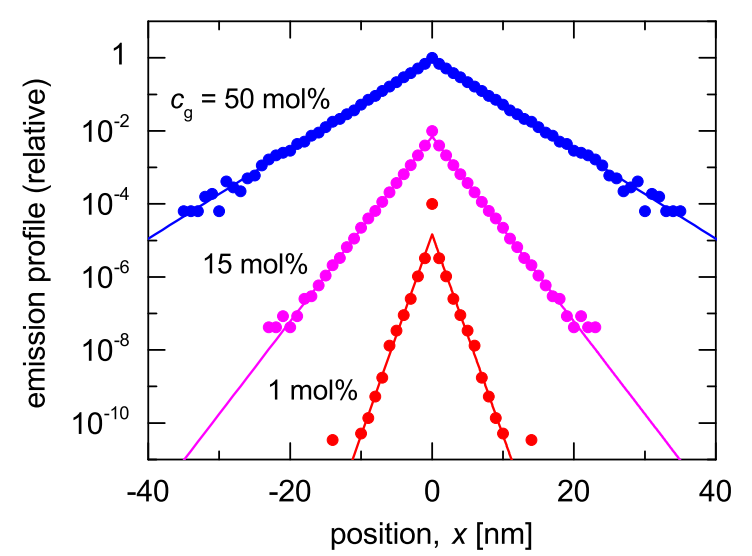

FIG. 7. Relative emission profiles (symmetrized) as obtained from KMC simulations within which absorption at random guest sites in the $x=0$ plane and subsequent Förster-type exciton diffusion with $R_{\mathrm{F}, \text { diff }}=1.5 \mathrm{~nm}$ takes place, for host-guest systems with guest concentrations of 1,15 , and $50 \mathrm{~mol} \%$.

significantly underestimates the contributions to diffusion via percolating pathways made available due to the randomness of the distribution of emitter molecule locations. In Sec. SIV in Ref. [19], an analogous expression for the case of Dexter transfer is given.

KMC simulation method. For determining the exciton diffusion coefficient from KMC simulations, we have used the simulation tool BUMBLEBEE [22] to mimic a photoluminescence (PL) experiment in which excitons are randomly absorbed at guest sites in a monolayer at $x=0$ in a simple cubic lattice. The excitons are allowed to diffuse and to decay radiatively, with a radiative lifetime $\tau=1 \mu \mathrm{s}$. The lattice constant is $a_{0}=1 \mathrm{~nm}$. From the continuum diffusion equation, the volume density of excitons resulting from an initial areal density $n_{\mathrm{A}}$ in the $x=0$ plane is given by

$$
n(x, t)=\frac{n_{\mathrm{A}}}{\sqrt{4 \pi D t}} \exp \left(-\frac{x^{2}}{4 D t}\right) .
$$

The emission profile is then given by

$$
\begin{aligned}
I(x) & =\int_{0}^{\infty} \frac{1}{\tau} \exp \left(-\frac{t}{\tau}\right) \frac{n_{\mathrm{A}}}{\sqrt{4 \pi D t}} \exp \left(-\frac{x^{2}}{4 D t}\right) d t \\
& =\frac{n_{\mathrm{A}}}{\sqrt{4 D \tau}} \exp \left(-\frac{|x|}{\sqrt{D \tau}}\right) .
\end{aligned}
$$

Using this expression, we determine the diffusion coefficient from the slope of a linear fit to the symmetrized function $\log _{10}[(I(x)+I(-x)) / 2]$ for $x \geqslant a_{0}$.

As an example, Fig. 7 shows the simulation results for the case of Förster-type diffusion with $R_{\mathrm{F} \text {,diff }}=1.5 \mathrm{~nm}$ in systems with guest concentrations of 1,15 , and $50 \mathrm{~mol} \%$, obtained from in total about $3 \times 10^{6}, 3 \times 10^{5}$, and $1 \times 10^{5}$ emission processes, respectively. Transfer over distances up to $6 \mathrm{~nm}$ in the $x, y$, and $z$ directions was included. No energetic disorder was assumed. The figure shows the relative number of emission processes per layer, after symmetrization and after shifting (for clarity) the $15 \mathrm{~mol} \%$ and $1 \mathrm{~mol} \%$ data by two and four orders of magnitude, respectively. The diffusion length, defined as $\sqrt{D \tau}$, was found to be $0.78,1.72$, and $3.54 \mathrm{~nm}$ 


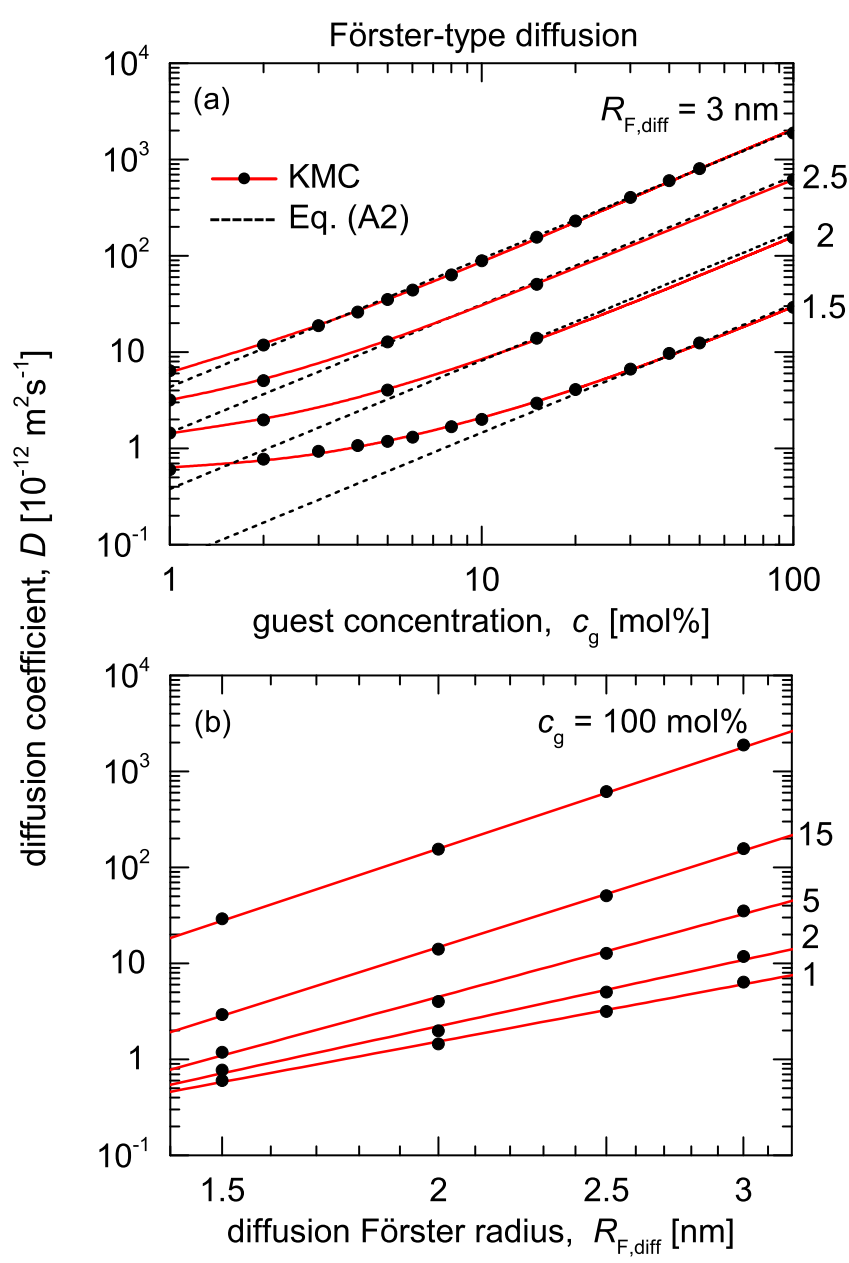

FIG. 8. KMC simulation results of the exciton diffusion coefficient in host-guest systems, resulting from Förster-type transfer with $\tau=1 \mu \mathrm{s}$. (a) Guest concentration dependence for various values of the Förster radius. The dashed lines indicate the result expected from Eq. (A2). (b) Dependence on the Förster radius, for various values of the guest concentration. The full red curves are a guide-to-the-eye.

for the 1, 15, and $50 \mathrm{~mol} \%$ systems, respectively. For Förstertype diffusion, $D \propto 1 / \tau$, so that the diffusion length does not depend on $\tau$. Interestingly, the figure reveals at $x=0$ a clear deviation from the continuum theory developed above: when the diffusion length is close to the intermolecular distance of $1 \mathrm{~nm}$, or smaller, a larger-than-expected fraction of the excitons does not diffuse. For nondilute systems (100 mol\% guest), there is no anomaly at $x=0$. The effect is due to contributions to the emission from excitons created at sites that are quite isolated from other guest sites. At very small $c_{\mathrm{g}}$, the concept of a diffusion coefficient is thus of limited value, even though it is possible to formally derive a value from the data for $x \geqslant a_{0}$ using the procedure discussed above. This should also be kept in mind when designing experiments probing exciton diffusion. When studying disordered systems, the view on the validity of the diffusion coefficient concept offered by this method is a clear advantage with respect to calculations of $D$ based on the mean-square of the diffusion distance.

KMC simulation results for Förster-type diffusion. Figure 8 shows the dependence of the exciton diffusion coefficient in host-guest systems resulting from Förster-type guest-guest transfer with $\tau=1 \mu \mathrm{s}$, as obtained from KMC simulations. When $D=1 \times 10^{-12} \mathrm{~m}^{2} \mathrm{~s}^{-1}$, the diffusion length is equal to the intersite distance of $1 \mathrm{~nm}$.

Panel (a) shows that Eq. (A2) is well obeyed for systems within which $c_{\mathrm{g}}$ and $R_{\mathrm{F} \text {,diff }}$ are sufficiently large, so that $D>10^{-11} \mathrm{~m}^{2} \mathrm{~s}^{-1}$. In such a situation, $D \propto c_{\mathrm{g}}{ }^{4 / 3}$. However, for situations that are more realistic for phosphorescent hostguest systems used in OLEDs, with $1 \mathrm{~mol} \%<c_{\mathrm{g}}<10 \mathrm{~mol} \%$ and $R_{\mathrm{F} \text {,diff }} \approx 2 \mathrm{~nm}$, a linear relationship $\left(D \propto c_{\mathrm{g}}\right)$ is a better approximation. The figure, furthermore, shows that the dependence of $D$ on $R_{\mathrm{F}, \text { diff }}$ decreases with decreasing $c_{\mathrm{g}}$. Panel (b) shows that for all concentrations a power-law dependence of the type $D \propto R_{\mathrm{F}, \mathrm{diff}}{ }^{\alpha}$ provides a good fit, with an exponent $\alpha$ that decreases from 6 for nondiluted systems to about 3.4, $3.9,4.9$, and 5.7 for $c_{\mathrm{g}}=1,2,5$, and $15 \mathrm{~mol} \%$, respectively. In the main text, these approximate descriptions of the dependence of $D$ on $c_{\mathrm{g}}$ and $R_{\mathrm{F}, \mathrm{diff}}$ will be used to provide a quite accurate description of the diffusion contribution to the TTA rate coefficient [Eq. (8)]. Figure S2 in Sec. SV in Ref. [19] provides analogous simulation results for the case of Dexter-type diffusion.
[1] C. Adachi, M. A. Baldo, S. Forrest, and M. Thompson, Appl. Phys. Lett. 77, 904 (2000).

[2] M. A. Baldo, C. Adachi, and S. R. Forrest, Phys. Rev. B 62, 10967 (2000).

[3] C. Murawski, K. Leo, and M. C. Gather, Adv. Mater. 25, 6801 (2013).

[4] N. C. Giebink, B. W. D’Andrade, M. S. Weaver, J. J. Brown, and S. R. Forrest, J. Appl. Phys. 105, 124514 (2009).

[5] S. Scholz, D. Kondakov, B. Lüssem, and K. Leo, Chem. Rev. 115, 8449 (2015).

[6] R. Coehoorn, H. van Eersel, P. Bobbert, and R. Janssen, Adv. Funct. Mater. 25, 2024 (2015).

[7] V. Rühle, A. Lukyanov, F. May, M. Schrader, T. Vehoff, J. Kirkpatrick, B. Baumeier, and D. Andrienko, J. Chem. Theory Comput. 7, 3335 (2011).
[8] P. Friederich, V. Meded, A. Poschlad, T. Neumann, V. Rodin, V. Stehr, F. Symalla, D. Danilov, G. Lüdemann, R. F. Fink, I. Kondov, F. von Wrochem, and W. Wenzel, Adv. Funct. Mater. 26, 5757 (2016).

[9] X. de Vries, P. Friederich, W. Wenzel, R. Coehoorn, and P. A. Bobbert, Phys. Rev. B 97, 075203 (2018).

[10] A. Miller and E. Abrahams, Phys. Rev. 120, 745 (1960).

[11] R. A. Marcus, Rev. Mod. Phys. 65, 599 (1993).

[12] H. van Eersel, P. A. Bobbert, and R. Coehoorn, J. Appl. Phys. 117, 115502 (2015).

[13] R. Coehoorn, L. Zhang, P. A. Bobbert, and H. van Eersel, Phys. Rev. B 95, 134202 (2017).

[14] R. Coehoorn, P. A. Bobbert, and H. van Eersel, Phys. Rev. B 96, 184203 (2017). 
[15] Y. Zhang and S. R. Forrest, Chem. Phys. Lett. 590, 106 (2013).

[16] L. Zhang, H. van Eersel, P. A. Bobbert, and R. Coehoorn, Chem. Phys. Lett. 662, 221 (2016).

[17] L. Zhang, H. van Eersel, P. A. Bobbert, and R. Coehoorn, Chem. Phys. Lett. 652, 142 (2016).

[18] A. Ligthart, X. de Vries, L. Zhang, M. C. W. M. Pols, P. A. Bobbert, H. van Eersel, and R. Coehoorn, Adv. Funct. Mater. 28, 1804618 (2018).

[19] See Supplemental Material at http://link.aps.org/supplemental/ 10.1103/PhysRevB.99.024201 for a brief description of the method used for deducing the TTA rate coefficients from KMC simulation results and experimental data, a tabulated overview of the KMC simulation data, and an analysis of exciton diffusion and TTA in the case of Dexter transfer.
[20] D. L. Dexter, J. Chem. Phys. 21, 836 (1953).

[21] X. de Vries, P. Friederich, W. Wenzel, R. Coehoorn, and P. A. Bobbert (unpublished).

[22] The BUMBLEBEE software is provided by Simbeyond B.V. (http: //simbeyond.com).

[23] K. B. Eisenthal and S. Siegel, J. Chem. Phys. 41, 652 (1964).

[24] A. Dogariu, D. Vacar, and A. J. Heeger, Phys. Rev. B 58, 10218 (1998).

[25] E. Engel, K. Leo, and M. Hoffmann, Chem. Phys. 325, 170 (2006).

[26] G. Lanzani, The Photophysics behind Photovoltaics and Photonics (Wiley-VCH Verlag GmbH \& Co. KGaA, Weinheim, Germany, 2012), p. 102.

[27] T. Förster, Z. Naturf. A 4, 321 (1949).

[28] T. Förster, Ann. Phys. 6, 55 (1948). 